\title{
Inverse dynamics and trajectory tracking control of a new six degrees of freedom spatial 3-RPRS parallel manipulator
}

\author{
Santhakumar Mohan ${ }^{1}$ and Burkhard Corves ${ }^{2}$ \\ ${ }^{1}$ Discipline of Mechanical Engineering, Indian Institute of Technology, Indore, 453552, India \\ ${ }^{2}$ Department of Mechanism Theory and Dynamics of Machines, RWTH Aachen University, \\ Aachen, 52072, Germany \\ Correspondence to: Santhakumar Mohan (santhakumar@iiti.ac.in)
}

Received: 25 April 2017 - Accepted: 9 July - Published: 28 July 2017

\begin{abstract}
This paper presents the complete dynamic model of a new six degrees of freedom (DOF) spatial 3-RPRS parallel manipulator. The geometry parameters of the manipulator are optimized for a given constant orientation workspace. Further, a robust task-space trajectory tracking control is also designed for the manipulator along with a nonlinear disturbance observer. To demonstrate the efficacy and show the complete performance of the proposed controller, virtual prototype experiments are executed using one of the multibody dynamics software namely MSC Adams. The computer-based virtual prototype experiment results show that the manipulator tracking performance is satisfactory with the proposed control scheme. In addition, the controller parameter sensitivity and robustness analyses are also accomplished.
\end{abstract}

\section{Introduction}

Parallel manipulators or parallel kinematic machines (PKMs) have fascinated a lot of research considerations in the past few decades for their greater performance over their serial complements, in terms of load-carrying capability, rigidity and accuracy (Merlet, 2000). Over the last few years, several parallel manipulators have matured from laboratory models to marketable devices. Unquestionably, the most successful manipulator is the Stewart-Gough platform manipulator. However, this manipulator has an extremely complex kinematics and coupled dynamics, and its design for a definite application remains a challenge for the researchers. Therefore, several manipulators varying in their number of degrees of freedom (DOF) from three to higher numbers (even redundant manipulators) have been proposed in the literature (Dasgupta and Mruthyunjaya, 2000; Merlet, 2000). Abundant 6DOF parallel robot configurations have been proposed in the literature (Merlet, 2000). While kinematically, the number of possible configurations is limited, the number of methods for applying them is fundamentally unbounded. In addition, distinct arrangements of the joints and legs may lead to very simplified direct kinematics, higher stiffness, or higher resolution. Most examples of 6-DOF fully-parallel manipulators may be classified by the type of their six identical serial chains being RRPS, RRRS, or PRRS (Bonev, 1998; Briot et al., 2009; Carbonari et al., 2013; Isaksson et al., 2012; Liu et al., 2002; Merlet, 2000; Pierrot, 1990; Uchiyama, 1993; Zhang, 2010). In this representation, RR stands for a universal joint (U), sometimes PR stands for a cylindrical joint (C), $\mathrm{P}$ for a prismatic joint, $\mathrm{R}$ for a rotary joint, $\mathrm{S}$ for a spherical joint, and an underlined letter designates an actuated joint. Note that the actuated/powered joint may be anyone in the chain but it is beneficial to place the actuators near the base, thus, reducing the inertia of the mobile platform or moving parts. There also exist configurations with only three chains, with two actuators per chain, as well as with various other combinations of chains. Examples are the prototype of Alizade and Tagiyev (1994), the "Eclipse" manipulator in Ryu et al. (1998), the mini-manipulator of Tahmasebi and Tsai (1994) (of type 3-PPSR), the robot of Byun and Cho (1997) (of type 3-PPSP), the manipulator of Nguyen et al. (2015) (of type of 3-PRRS/3-CRS) and several others. These manipulators are in general easier to evaluate but have 

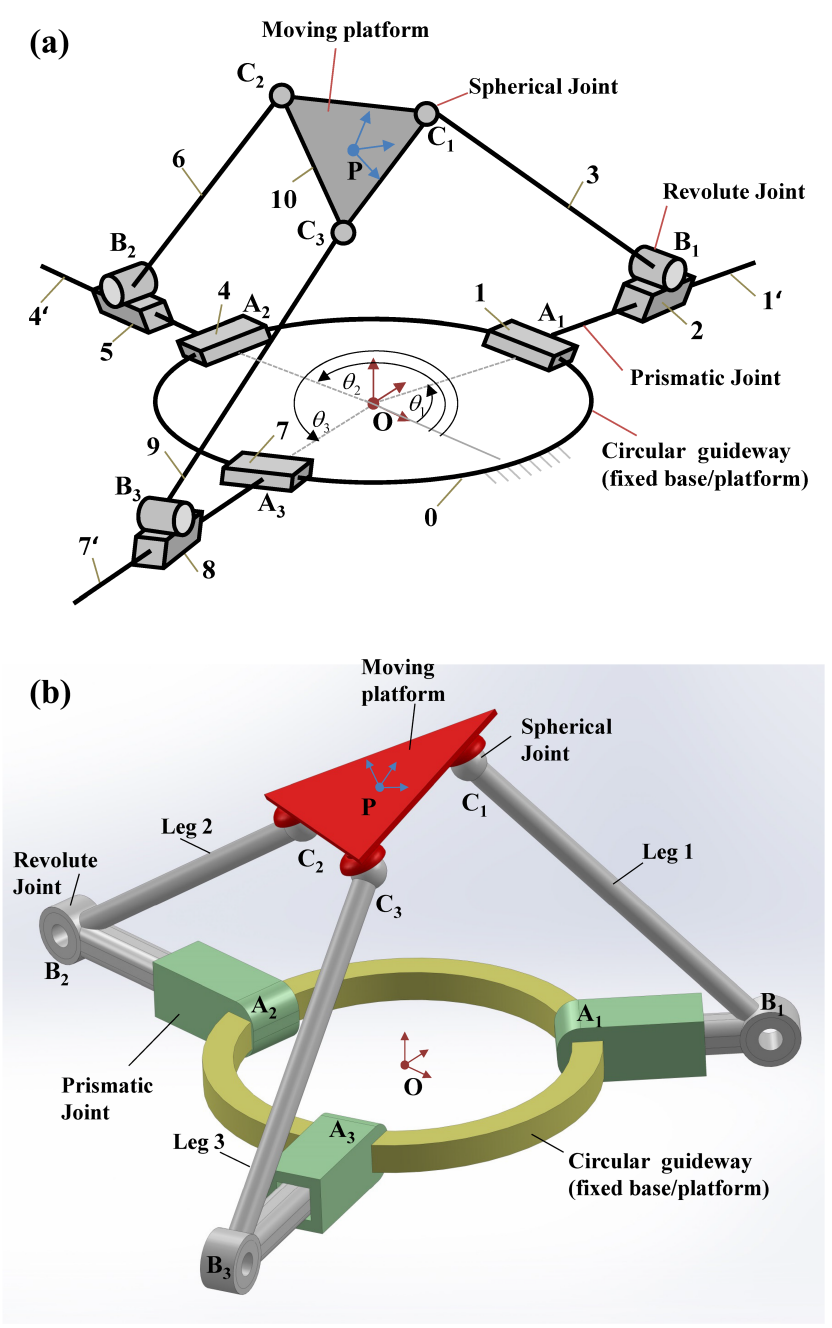

Figure 1. (a) Kinematic arrangement. (b) Solid (3-D) model of the manipulator. Conceptual design of the proposed manipulator.

a lower overall stiffness since only three kinematic chains support the mobile platform.

In this direction, a new 6-DOF spatial 3-RPRS parallel manipulator was introduced in Venkatesan et al. (2014). The manipulator has three legs mounted on a circular guide at the base, which allows it to exhibit large (i.e., kinematically unbounded) yaw motions, which is not very common in platform-type spatial parallel manipulators. In Venkatesan et al. (2014), only the inverse kinematic analysis of the manipulator was presented. The forward kinematic problem of the same manipulator was introduced in Nag et al. (2017), wherein the solution procedure was elaborated using a numerical example. In the present paper, the inverse dynamic model and trajectory tracking control of the 3-RPRS manipulator is studied comprehensively. The main advantage of the 3-RPRS manipulator is with respect to their dynamics and simplified kinematics. As the actuators being usually the heavy part of a manipulator are fixed at the base, the mobile part of the robot is reduced to the three legs and the mobile platform. Consequently, higher velocities and accelerations of the mobile platform can be achieved. Another benefit is that the legs are made of only thin rods, thus, reducing the risk of leg interference. Further, the geometrical/physical parameters of the manipulator are also optimized for a given constant orientation workspace. The inverse dynamic model is obtained using the Lagrangian dynamic formulation method (Abdellatif and Heimann, 2009). The proposed robust task-space trajectory tracking controller is based on a centralized proportional-integral-derivative (PID) control along with a nonlinear disturbance observer. The control schemes for parallel manipulator may be principally separated into two types, joint-space control established in joint-space coordinates (Davliakos and Papadopoulos, 2008; Honegger et al., 2000; Kim et al., 2000; Nguyen et al., 1992; Yang et al., 2010), and task-space control designed based on the task-space coordinates (Kim et al., 2005; Ting et al., 2004; $\mathrm{Wu}$ and $\mathrm{Gu}, 2005)$. The joint-space control approach can be readily employed as an assemblage of several independent single-input single-output (SISO) control systems using the data on each actuator feedback only. A classical PID control in joint-space along with gravity compensation has been employed in industry, but it does not always assure a great performance for parallel manipulators. However, the proposed robust task-space control approach improves the overall control performance by rejecting the uncertainty and nonlinear effects in motion equations. The rejections of system or model uncertainty, unknown external disturbance and nonlinear effects in the system motions have been completed in the proposed control scheme with the help of an equivalent control law; a feed-forward control scheme and a nonlinear disturbance observer along with the nonlinear PID control scheme. In the proposed task-space control method, the desired motion of the end effector in task-space is used directly as the reference input of the control scheme. That is, the motion of the end effector can be obtained from the system sensors and compared with the reference input to form a feedback error in task-space. Therefore, an exact kinematics model is not required in the task-space control, and thus this method is sensitive to joint-space errors or end effector pose errors due to joint clearances and other mechanical inaccuracies. The validity of the proposed control scheme is demonstrated with the help of virtual prototype experiments. The performance of the proposed control scheme including closed-loop stability, precision, sensitivity and robustness is analysed in theory and simulation.

To this end, this paper is organized as follows: Sect. 2 presents the system description and mathematical background of the manipulator on how the dynamic model for a parallel manipulator is developed using the LagrangianEuler formulation. Section 3 presents the proposed robust trajectory tracking control scheme in task space along with its stability proof done based on Lyapunov's method. Section 4 summarizes and discusses the performance evaluation 


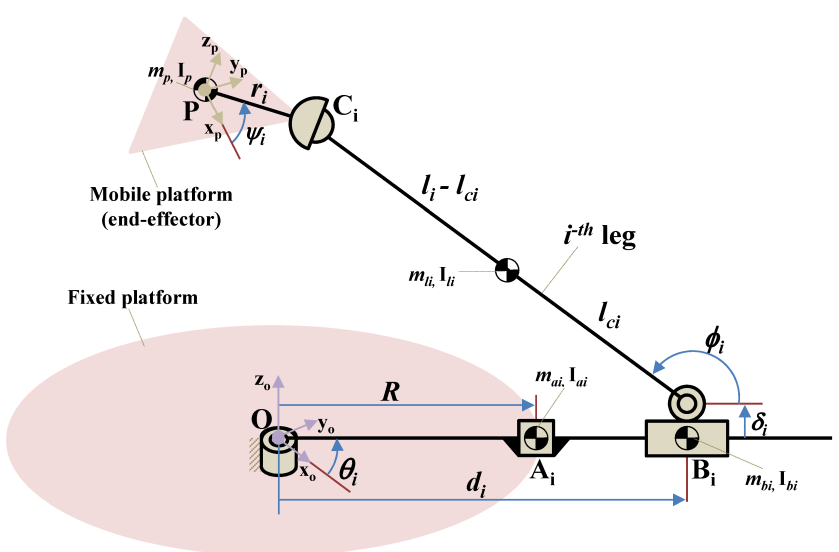

Figure 2. Kinematic arrangement of $i$ th leg of the proposed manipulator.

of the proposed control scheme and the robustness analysis. Finally, the conclusions are drawn.

\section{System description and mathematical background}

The proposed manipulator consists of three legs or kinematic chains and each with a rotary-prismatic-rotary-spherical (RPRS) configuration as shown in Fig. 1. The base of the platform (fixed) has a circular guide on which three sliders glide, all are actuated by rotary joints at the centre of the base platform. The active prismatic joint is situated on each guideway connecting the central rotary joint and the slider on the circular guide rail and on actuation, the prismatic joints move radially. There is a passive link which is connected with the slider block on the prismatic joint through a revolute joint and with the end-effector (mobile platform) through the help of a spherical joint. In all three legs, the starting rotary joint and the prismatic joint are actuated and other joints namely rotary and spherical joints are passive. The geometry of the manipulator is identical to the one reported in Venkatesan et al. (2014).

The kinematic arrangement along with the centre of mass locations of the $i$ th leg of the proposed manipulator is presented in Fig. 2. For the purpose of kinematic analysis, a first coordinate system $O\left(x_{o}, y_{o}, z_{o}\right)$ is fixed to the fixed base platform and a second coordinate system $P\left(x_{p}, y_{p}, z_{p}\right)$ is attached to the moving base platform (end-effector) as shown in Figs. 1 and 2. The transformation from the moving platform to the fixed base can be described using the position vector $\boldsymbol{P}_{P}^{O}$ and the rotation matrix ${ }_{P}^{O} \mathbf{R}$ of the moving platform with respect to the fixed base platform, and are given as:

$\boldsymbol{P}_{P}^{O}=\left[\begin{array}{lll}P_{x} & P_{y} & P_{z}\end{array}\right]^{T}$
${ }_{P}^{O} \mathbf{R}=$

$\left[\begin{array}{ccc}\cos \alpha \cos \beta & \cos \alpha \sin \beta \sin \gamma-\sin \alpha \cos \gamma & \cos \alpha \sin \beta \cos \gamma+\sin \alpha \sin \gamma \\ \sin \alpha \cos \beta & \sin \alpha \sin \beta \sin \gamma+\cos \alpha \cos \gamma & \sin \alpha \sin \beta \cos \gamma-\cos \alpha \sin \gamma \\ -\sin \beta & \cos \beta \sin \gamma & \cos \beta \cos \gamma\end{array}\right]$

where, $P_{x}, P_{y}$ and $P_{z}$ are the positions of the end-effector and $\alpha, \beta$ and $\gamma$ are the roll, pitch and yaw angles of the end-effector with respect to the fixed base platform coordinate system. The end-effector positions and orientations are considered as the task-space displacement variables and the task-space displacement vector is denoted as: $\boldsymbol{\mu}=\left[\begin{array}{llllll}P_{x} & P_{y} & P_{z} & \alpha & \beta & \gamma\end{array}\right]^{T}$.

The vector of actuator coordinates (joint-space displacements) namely rotation angles and translation displacements of the manipulator are denoted as: $\boldsymbol{q}=$ $\left[\begin{array}{llllll}\theta_{1} & \theta_{2} & \theta_{3} & d_{1} & d_{2} & d_{3}\end{array}\right]^{T}$.

The position coordinates of the spherical joints on the mobile platform with respect to the fixed coordinate system $\mathbf{C}_{i}=\left[\begin{array}{lll}C_{x i} & C_{y i} & C_{z i}\end{array}\right]^{T}$ can be derived as follows:

$\mathbf{C}_{i}^{O}=\mathbf{C}_{i}={ }_{P}^{O} \mathbf{R C}_{i}^{P}+\boldsymbol{P}_{P}^{O}$

where, $\mathbf{C}_{i}^{P}$ is the position vector of the $i$ th spherical joint with respect to the mobile coordinate system $P\left(x_{p}, y_{p}, z_{p}\right)$ and this vector depends on the distance $r_{i}$ and an angle $\psi_{i}$ (fixed geometry variables of the manipulator). $i$ is the corresponding leg number and $i=1,2,3$. These spherical joint position coordinates $\mathbf{C}_{i}$ can be used to establish the joint-space variables namely $\theta_{i}$ and $d_{i}$, as follows:

$\theta_{i}=a \tan 2\left(C_{y i}, C_{x i}\right)$

$d_{i}=\sqrt{l_{i}^{2}-\left(C_{z i}-\delta_{i}\right)^{2}}+\sqrt{C_{x i}^{2}+C_{y i}^{2}}$

Further, using Eqs. (4) and (5), the position coordinates of the points $\boldsymbol{A}_{i}$ and $\boldsymbol{B}_{i}$ representing circular and linear slider block locations can be obtained as follows:

$$
\begin{aligned}
\boldsymbol{A}_{\mathrm{i}} & =\left[\begin{array}{lll}
R \cos \theta_{i} & R \sin \theta_{i} & 0
\end{array}\right]^{T} \\
\boldsymbol{B}_{\mathrm{i}} & =\left[\begin{array}{lll}
d_{i} \cos \theta_{i} & d_{i} \sin \theta_{i} & 0
\end{array}\right]^{T}
\end{aligned}
$$

The forward kinematic model of this manipulator can be obtained with the help of loop-closure equations and forward kinematic univariate (Nag et al., 2017). The inverse kinematic solutions of the proposed manipulator are similar to the reported one in Venkatesan et al. (2014).

The velocity and acceleration relations of the manipulator can be obtained with the help of the inverse Jacobian matrix, as given:

$$
\begin{aligned}
\dot{\boldsymbol{q}} & =\mathbf{J}(\mu) \dot{\boldsymbol{\mu}} \\
\ddot{\boldsymbol{q}} & =\mathbf{J}(\mu) \ddot{\boldsymbol{\mu}}+\dot{\mathbf{J}}(\mu) \dot{\boldsymbol{\mu}}
\end{aligned}
$$

where, $\dot{\boldsymbol{q}} \in \mathfrak{R}^{6 \times 1}$ is the vector of joint-space velocities, $\dot{\boldsymbol{\mu}} \in$ $\Re^{6 \times 1}$ is the vector of task-space velocities and $\mathbf{J}(\mu) \in \Re^{6 \times 6}$ is the inverse Jacobian matrix of the manipulator. $\ddot{\boldsymbol{q}} \in \mathfrak{R}^{6 \times 1}$ is 


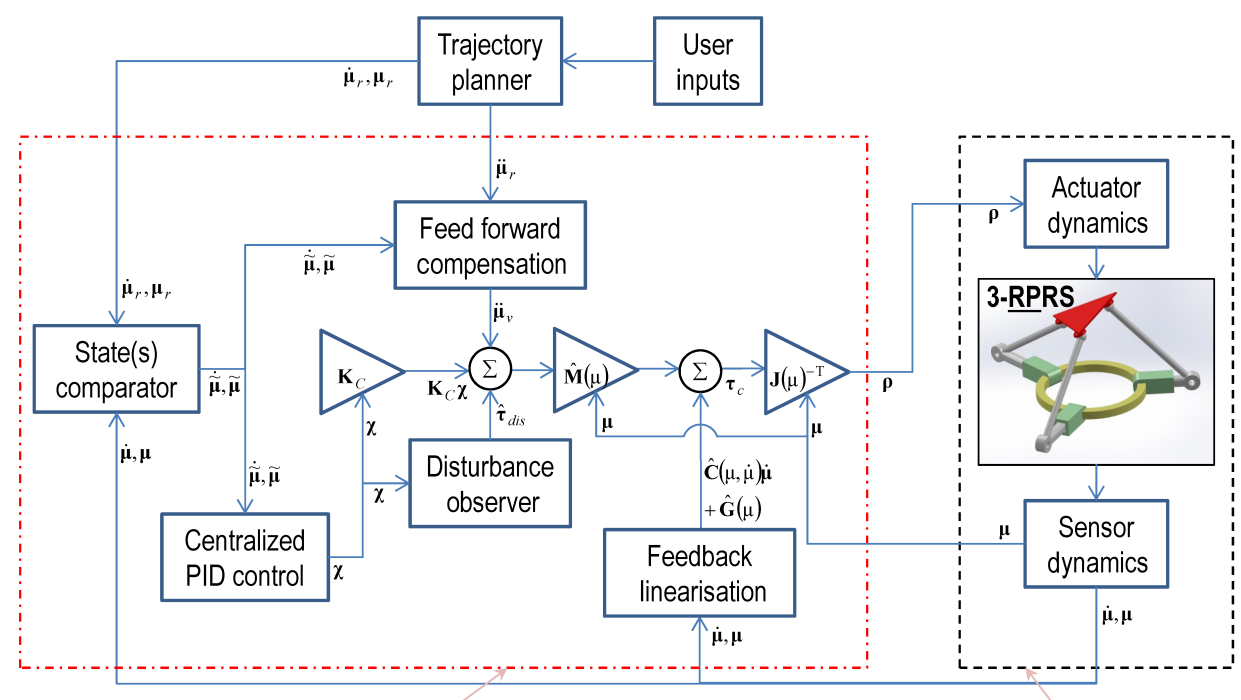

Trajectory tracking control scheme

Robotic system

Figure 3. Block diagram representation of the proposed robust task-space control scheme.

the vector of joint-space accelerations, $\ddot{\mu} \in \Re^{6 \times 1}$ is the vector of task-space accelerations and $\mathbf{J}(\mu) \in \mathfrak{R}^{6 \times 6}$ is the time derivative of the inverse Jacobian matrix of the manipulator.

Understanding the manipulator dynamics and, the relationship between the joint forces/moments and their effect on the joint parameters are essential in designing the system as it communicates the effect of the driving efforts on the end effector. The method adopted for the formulation of the dynamic model is Euler-Lagrange (second kind) formulation which is based on the total energy of the system and adopted because of its simplicity. Since the proposed system is considered as a rigid body system, the total energy of the system is only the sum of kinetic and potential energies of the individual moving components of the system. The kinematic arrangement of the mechanism along with locations of the center of masses of bodies is presented in Fig. 2. The proposed manipulator consists of eleven bodies including the fixed platform. Each leg has three moving masses/bodies and the end-effector (refer Fig. 1). The kinetic energy and potential energy of the manipulator corresponds to the sum of kinetic energies and sum of potential energies of these individual moving components of the manipulator, respectively, which are given as follows:

$$
\begin{aligned}
\mathrm{KE} & =\frac{1}{2} \sum_{j=1}^{10}\left(m_{j}\left(\dot{x}_{\mathrm{cm} j}^{2}+\dot{y}_{\mathrm{cm} j}^{2}+\dot{z}_{\mathrm{cm} j}^{2}\right)+\mathbf{I}_{j} \boldsymbol{\omega}_{j}^{2}\right) \\
\mathrm{PE} & =\sum_{j=1}^{10} m_{j} g z_{j}
\end{aligned}
$$

where, KE and PE are the total sum of the kinetic and potential energy of the mechanism. $m_{j}$ and $\mathbf{I}_{j}$ are the corresponding mass and inertia matrix of the $j$ th link/body, respectively.
Similarly, $x_{\mathrm{cm} j}, y_{\mathrm{cm} j}$ and $z_{\mathrm{cm} j}$ are the locations of the center of mass of the $j$ th link/body, respectively. $\omega_{j}$ is the vector of angular velocities of the $j$ th link/body, $\dot{x}_{\mathrm{cm} j}, \dot{y}_{\mathrm{cm} j}$ and $\dot{z}_{\mathrm{cm} j}$ are the linear velocities of the centre of mass of the $j$ th link/body, respectively. $g$ is the gravity constant.

The Lagrange equation as per the formulation is given by

$L_{\text {mechanism }}=\mathrm{KE}-\mathrm{PE}$

$\tau_{i}=\frac{d}{\mathrm{~d} t}\left(\frac{\partial L_{\text {mechanism }}}{\partial \dot{\mu}_{i}}\right)-\frac{\partial L_{\text {mechanism }}}{\partial \mu_{i}}$

where $L_{\text {mechanism }}$ is the Lagrangian of the mechanism and $\tau_{i}$ is the corresponding task-space force/torque which includes the control inputs and all other non-conservative external effects. $\mu_{i}$ and $\dot{\mu}_{i}$ are the corresponding task-space displacement and velocity, respectively.

The dynamic equations of motion of the proposed mechanism in task-space are obtained with the help of above relations, and they can be represented in matrix form as follows:

$\mathbf{M}(\mu) \ddot{\boldsymbol{\mu}}+\mathbf{C}(\mu, \dot{\mu}) \dot{\boldsymbol{\mu}}+\boldsymbol{g}(\mu)=\boldsymbol{\tau}$

where $\ddot{\mu} \in \mathfrak{R}^{6 \times 1}$ is the vector of task-space accelerations. $\mathbf{M}(\mu) \in \mathfrak{R}^{6 \times 6}$ is the inertia matrix; $\mathbf{C}(\mu, \dot{\mu}) \in \mathfrak{R}^{6 \times 6}$ is the centripetal and Coriolis force matrix; $\boldsymbol{g}(\mu) \in \mathfrak{R}^{6 \times 1}$ is the gravitational force vector; $\tau \in \mathfrak{R}^{6 \times 1}$ is the input vector. Since, the input vector consists of control inputs and other non-conservative effects, the equation of motion can be rewritten by considering the non-conservatives effects as follows:

$\mathbf{M}(\mu) \ddot{\boldsymbol{\mu}}+\mathbf{C}(\mu, \dot{\mu}) \dot{\boldsymbol{\mu}}+\boldsymbol{f}(\mu, \dot{\mu})+\boldsymbol{g}(\mu)=\boldsymbol{\tau}_{\mathrm{c}}+\boldsymbol{\tau}_{\mathrm{d}}$

where $\boldsymbol{f}(\mu, \dot{\mu}) \in \mathfrak{R}^{6 \times 1}$ is the frictional force vector; $\boldsymbol{\tau}_{\mathrm{c}} \in$ $\Re^{6 \times 1}$ is the control (actuator) input vector in task-space; $\boldsymbol{\tau}_{\mathrm{d}} \in$ 
Table 1. Optimized geometrical design parameters of the manipulator

\begin{tabular}{|c|c|c|c|c|c|c|c|c|c|}
\hline \multicolumn{7}{|c|}{$\begin{array}{l}\text { Given work } \\
\text { volume }\end{array}$} & \multicolumn{3}{|c|}{$\begin{array}{c}\text { Geometrical design } \\
\text { parameters of the manipulator }\end{array}$} \\
\hline $\begin{array}{l}\text { Work } \\
\text { volume }\end{array}$ & $\begin{array}{l}x \text { a } \\
\lim \end{array}$ & & & & & & $\begin{array}{l}\text { End-effector } \\
\text { size }\end{array}$ & $\begin{array}{l}\text { Link length } \\
\text { (s) }\end{array}$ & $\begin{array}{l}\text { Maximum limit } \\
\text { of the linear actuators }\end{array}$ \\
\hline$V_{w}$ & $x_{\min }$ & $x_{\max }$ & $y_{\min }$ & $y_{\max }$ & $z_{\min }$ & $z_{\max }$ & $r_{i}$ & $l_{i}$ & $d_{i(\max )}$ \\
\hline $0.008 \mathrm{~m}^{3}$ & $-0.1 \mathrm{~m}$ & $0.1 \mathrm{~m}$ & $-0.1 \mathrm{~m}$ & $0.1 \mathrm{~m}$ & $0.05 \mathrm{~m}$ & $0.25 \mathrm{~m}$ & $0.098 \mathrm{~m}$ & $0.277 \mathrm{~m}$ & $0.559 \mathrm{~m}$ \\
\hline $0.027 \mathrm{~m}^{3}$ & $-0.15 \mathrm{~m}$ & $0.15 \mathrm{~m}$ & $-0.15 \mathrm{~m}$ & $0.15 \mathrm{~m}$ & $0.05 \mathrm{~m}$ & $0.35 \mathrm{~m}$ & $0.081 \mathrm{~m}$ & $0.382 \mathrm{~m}$ & $0.699 \mathrm{~m}$ \\
\hline $0.064 \mathrm{~m}^{3}$ & $-0.20 \mathrm{~m}$ & $0.20 \mathrm{~m}$ & $-0.20 \mathrm{~m}$ & $0.20 \mathrm{~m}$ & $0.05 \mathrm{~m}$ & $0.45 \mathrm{~m}$ & $0.064 \mathrm{~m}$ & $0.514 \mathrm{~m}$ & $0.884 \mathrm{~m}$ \\
\hline
\end{tabular}

Table 2. Physical parameters of the proposed manipulator

\begin{tabular}{llll}
\hline Parameter & Value & Parameter & Value \\
\hline$m_{a i}$ & $1.5 \mathrm{~kg}$ & $m_{l i}$ & $0.5 \mathrm{~kg}$ \\
$m_{b i}$ & $0.7 \mathrm{~kg}$ & $m_{p}$ & $1.2 \mathrm{~kg}$ \\
$I_{a i}$ & $0.07 \mathrm{kgm}^{2}$ & $I_{b i}$ & $0.36 \mathrm{kgm}^{2}$ \\
$I_{l i}$ & $0.12 \mathrm{kgm}^{2}$ & $I_{p x}$ & $0.2 \mathrm{kgm}^{2}$ \\
$I_{p y}$ & $0.02 \mathrm{kgm}^{2}$ & $I_{p z}$ & $0.03 \mathrm{kgm}^{2}$ \\
$r_{i}$ & $0.064 \mathrm{~m}$ & $l_{i}$ & $0.514 \mathrm{~m}$ \\
\hline
\end{tabular}

$\mathfrak{R}^{6 \times 1}$ is the disturbance vector in task-space (which includes external disturbances and internal uncertainties namely parameter variations, noises, etc.). The actual disturbance vector $\left(\boldsymbol{\tau}_{\mathrm{d}}\right)$ can be expressed as follows:

$$
\begin{aligned}
& \boldsymbol{\tau}_{\mathrm{d}}=\boldsymbol{\tau}_{\mathrm{ed}}-(\Delta \mathbf{M}(\mu) \ddot{\boldsymbol{\mu}}+\Delta \mathbf{C}(\mu, \dot{\mu}) \dot{\boldsymbol{\mu}}+\Delta \boldsymbol{g}(\mu)) \\
& +\zeta+\tau_{\text {um }} \\
& \Delta \mathbf{M}(\mu)=\mathbf{M}(\mu)-\hat{\mathbf{M}}(\mu), \Delta \mathbf{C}(\mu, \dot{\mu})=\mathbf{C}(\mu, \dot{\mu}) \\
& -\hat{\mathbf{C}}(\mu, \dot{\mu}), \Delta \boldsymbol{g}(\mu)=\boldsymbol{g}(\mu)-\hat{\boldsymbol{g}}(\mu)
\end{aligned}
$$

where $\tau_{\text {ed }} \in \mathfrak{R}^{6 \times 1}$ is the vector of unknown external disturbances in task-space; $\zeta \in \mathfrak{R}^{6 \times 1}$ is the vector of process and measurement noises in task-space; $\boldsymbol{\tau}_{\text {um }} \in \mathfrak{R}^{6 \times 1}$ is the vector of un-modelled dynamic effects in task-space; $\hat{\mathbf{M}}(\mu)$, $\hat{\mathbf{C}}(\mu, \dot{\mu})$ and $\hat{\boldsymbol{g}}(\mu)$ are the known (inaccurate) values of the inertia matrix, centripetal and Coriolis force matrix and gravity vector, respectively.

Since the model is obtained in the task-space the control inputs in the joint-space can be expressed as follows:

$\rho=\mathbf{J}(\mu)^{-T} \boldsymbol{\tau}_{\mathrm{c}}$

where, $\rho \in \mathfrak{R}^{6 \times 1}$ is the vector of inputs (control inputs) in the joint-space; $\mathbf{J}(\mu)^{-T} \in \mathfrak{R}^{6 \times 6}$ is the transpose of the inverse Jacobian matrix of the manipulator. The obtained mathematical model that describes the dynamic behavior of the proposed manipulator is verified using a multi-body dynamics package namely MSC Adams.
Table 3. Controller parameters for the virtual prototype experiments.

\begin{tabular}{llll}
\hline Parameter & Value & Parameter & Value \\
\hline $\mathbf{K}$ & $10 \mathbf{I}_{6 \times 6}$ & $\boldsymbol{\Lambda}$ & $2 \mathbf{I}_{6 \times 6}$ \\
$\boldsymbol{\Gamma}$ & $10 \mathbf{I}_{6 \times 6}$ & 10 & $0.1 \mathrm{~s}$ \\
$\mathbf{K}_{P}$ & $100 \mathbf{I}_{6 \times 6}$ & $\mathbf{K}_{\mathrm{D}}$ & $20 \mathbf{I}_{6 \times 6}$ \\
\hline
\end{tabular}

\section{Robust task-space trajectory tracking control scheme}

In this section, a robust nonlinear controller along with a disturbance observer is proposed to track a given desired taskspace position (end-effector pose) trajectory of the manipulator. The proposed task-space control vector is given as follows:

$$
\begin{aligned}
& \boldsymbol{\tau}_{\mathrm{c}}=\hat{\mathbf{M}}(\mu)\left[\ddot{\boldsymbol{\mu}}_{\mathrm{v}}+\mathbf{K} \xi+\hat{\boldsymbol{\tau}}_{\mathrm{dis}}\right]+\hat{\mathbf{C}}(\mu, \dot{\mu}) \dot{\boldsymbol{\mu}}+\hat{\boldsymbol{g}}(\mu) \\
& \ddot{\boldsymbol{\mu}}_{\mathrm{v}}=\ddot{\boldsymbol{\mu}}_{r}+2 \boldsymbol{\Lambda} \dot{\tilde{\mu}}+\boldsymbol{\Lambda}^{2} \widetilde{\boldsymbol{\mu}} \\
& \boldsymbol{\xi}=\dot{\tilde{\boldsymbol{\mu}}}+2 \boldsymbol{\Lambda} \widetilde{\boldsymbol{\mu}}+\boldsymbol{\Lambda}^{2} \int \tilde{\boldsymbol{\mu}} \mathrm{d} t \\
& \hat{\boldsymbol{\eta}}=\boldsymbol{\Gamma} \int \xi \mathrm{d} t \\
& \boldsymbol{\eta}=\boldsymbol{\tau}_{\mathrm{d}}-\boldsymbol{f}(\mu, \dot{\mu}) \\
& \dot{\tilde{\boldsymbol{\mu}}}=\dot{\boldsymbol{\mu}}_{r}-\dot{\boldsymbol{\mu}} \\
& \widetilde{\boldsymbol{\mu}}=\boldsymbol{\mu}_{r}-\boldsymbol{\mu}
\end{aligned}
$$

where, $\mathbf{K} \in \mathfrak{R}^{6 \times 6}$ and $\boldsymbol{\Gamma} \in \mathfrak{R}^{6 \times 6}$ are the controller and observer gain matrices of the proposed controller, respectively and chosen as symmetric positive definite (SPD) matrices. $\ddot{\boldsymbol{\mu}}_{\mathrm{v}}$ is the virtual desired acceleration vector. $\ddot{\boldsymbol{\mu}}_{r}, \dot{\boldsymbol{\mu}}_{r}$ and $\boldsymbol{\mu}_{r}$ are the desired task-space acceleration, velocity and position vectors, respectively. $\tilde{\boldsymbol{\mu}}$ and $\dot{\tilde{\boldsymbol{\mu}}}$ are the vectors of task-space position errors and velocity errors, respectively. $\Lambda \in \mathfrak{R}^{6 \times 6}$ is the centralized PID control gain matrix of the proposed control scheme and chosen as a SPD matrix. $\xi \in \mathfrak{R}^{6 \times 1}$ is the centralized proportional-integral-derivative (PID) control input vector and it was obtained from the second order integral sliding mode control vector. $\hat{\eta} \in \mathfrak{R}^{6 \times 1}$ is the vector of estimated disturbances which includes the frictional effects of the manipulator. $\eta \in \mathfrak{R}^{6 \times 1}$ is the vector of lumped disturbances of the manipulator. 
Table 4. Performance comparison of the controllers

\begin{tabular}{lrr|rr}
\hline Control scheme & \multicolumn{2}{c}{ Root-mean-square (RMS) values of end-effector pose errors } \\
\cline { 2 - 4 } & \multicolumn{2}{c}{ Circular Path } & \multicolumn{2}{c}{ Polygon Path } \\
\cline { 2 - 4 } & $\kappa_{\text {rms }}$ in mm & $\varphi_{\text {rms }}$ in deg & \multirow{2}{*}{$\kappa_{\text {rms }}$ in mm } & $\varphi_{\text {rms }}$ in deg \\
\hline Computed torque control (CTC) & 3.02 & 0.33 & 4.49 & 0.04 \\
Proposed control without a disturbance observer & 0.84 & 0.18 & 0.99 & 0.02 \\
Proposed control with a disturbance observer & 0.43 & 0.05 & 0.54 & 0.01 \\
\hline
\end{tabular}

Figure 3 shows the block diagram representation of the proposed task-space trajectory tracking control scheme. As depicted in this figure, the block diagram flow starts with the desired task-space variables as a user input which is a function of time, $t$. The trajectory planner provides the desired task-space coordinates namely time trajectories of the task-space position, velocity, and acceleration vectors respectively, based on the user inputs. The sensor and actuator dynamics are also incorporated into the manipulator dynamical model. The state comparator gives the task-space tracking errors which act as an input to the proposed controller. The proposed control law can be mainly divided into four parts according to different functions. The first term is the feedforward dynamics compensation computed by inverting plant model, is responsible for reducing and eliminating tracking errors. The second term is the centralized PID control law which acts as a feedback part results in holding the stability of the whole system. The third term of the proposed control law is the disturbance estimation term based on the observer update law. This estimator estimates all the uncertainties including external disturbances and unknown nonlinear dynamics of the manipulator based on the perturbation from the dynamics of the PID controller. Therefore at each instant, the control input compensates for the uncertainty that exists during task-space trajectory tracking of the manipulator system. Finally, the feedback linearization of the nonlinear terms in the manipulator dynamics based on the known (inaccurate) values as a fourth term. Since manipulator actuators are actuated in the joint-space, the control vector is transformed to the joint space with the help of inverse Jacobian matrix transpose. The stability analysis of the proposed control law has been derived by the Lyapunov's direct method and explained in the following subsection.

\subsection{Stability analysis}

In this subsection, the Lyapunov's direct method is employed to show the asymptotic convergence nature of the proposed controller. The following assumptions are considered to ensure the asymptotic convergence of both disturbance estimation errors and tracking errors of the proposed close-loop system.
Assumption 1: The controller and estimator gain matrices namely $\mathbf{K}, \boldsymbol{\Gamma}$ and $\boldsymbol{\Lambda}$ are constant symmetric and positive definite matrices, by design, that is:

$$
\mathbf{K}=\mathbf{K}^{T}>0, \boldsymbol{\Gamma}=\boldsymbol{\Gamma}^{T}>0, \text { and } \boldsymbol{\Lambda}=\boldsymbol{\Lambda}^{T}>0
$$

Assumption 2: The rate of change of the disturbance acting on the manipulator is negligible in comparison with the estimated error dynamics, i.e., disturbances are slowly varying, $\dot{\eta} \approx 0$ and this assumption is not overly restrictive and is commonly made in the robot manipulator literature (Kelly et al., 2005). Moreover, the lumped disturbance vector $\eta$ is assumed to be bounded and there exists a constant $\eta_{\mathrm{U}}$ such that, $0 \leq|\boldsymbol{\eta}| \leq \boldsymbol{\eta}_{\mathrm{U}}$. But this value of upper bound is not required to be known for the controller design.

Theorem 1: Consider the equations of motion of the manipulator as given in Eq. (14), if the control input vector is chosen as defined in Eq. (18), then the taskspace tracking errors and observer errors converge to zero asymptotically.

Proof 1: Consider a positive Lyapunov's candidate function as:

$V=\frac{1}{2} \xi^{T} \xi+\frac{1}{2} \widetilde{\eta}^{T} \Gamma^{-1} \widetilde{\eta}$

where, $\widetilde{\eta}$ is the vector of the lumped disturbance estimation errors and is defined as follows:

$\widetilde{\eta}=\eta-\hat{\eta}$

Differentiating Eq. (21) with respect to time along with its state trajectories resulting into,

$\dot{V}=\xi^{T} \dot{\xi}+\widetilde{\eta}^{T} \Gamma^{-1} \dot{\tilde{\eta}}$ 


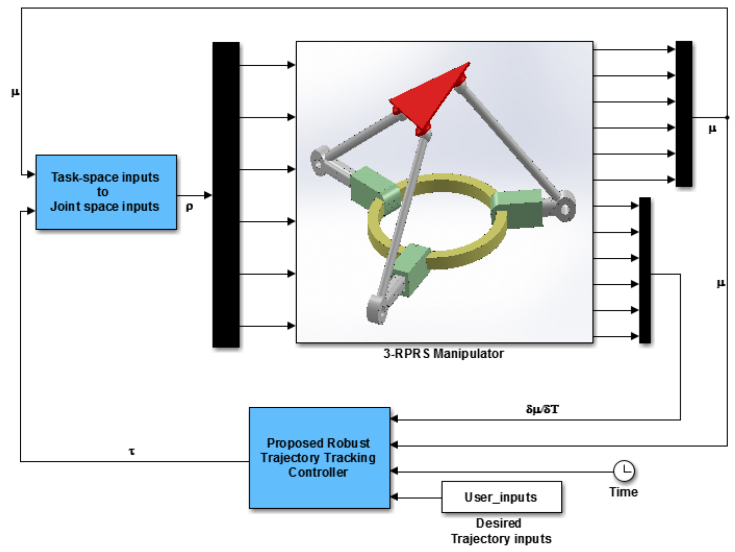

(a) Complete co-simulation model of the proposed system

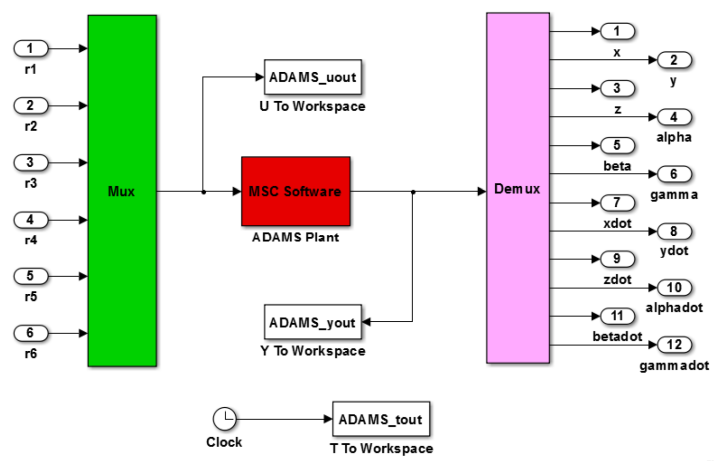

(b) 3-RPRS manipulator as a ADAMS plant in Simulink

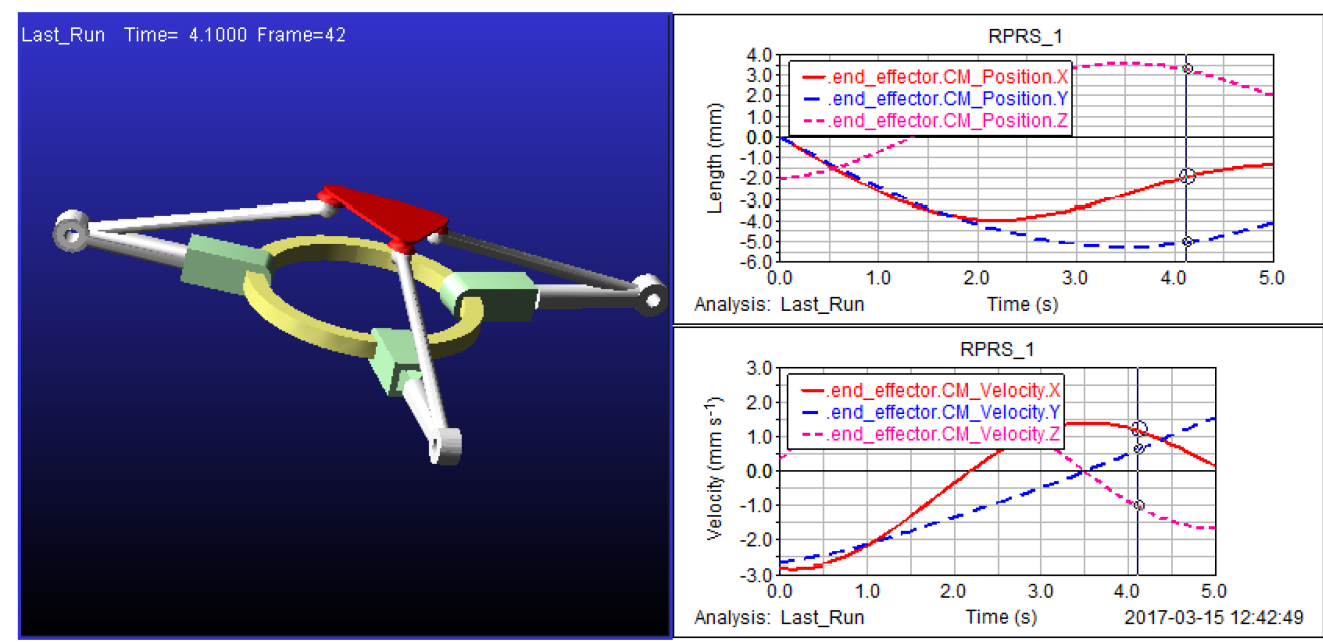

(c) 3-RPRS manipulator model in ADAMS

Figure 4. Block diagram representation of the proposed robust task-space control scheme.

where, $\dot{\xi}$ and $\dot{\tilde{\eta}}$ are the time derivatives of the centralized PID control vector and disturbance estimation errors, respectively, and these can be denoted as follows:

$$
\begin{aligned}
& \dot{\xi}=\ddot{\tilde{\mu}}+2 \boldsymbol{\Lambda} \dot{\tilde{\mu}}+\boldsymbol{\Lambda}^{2} \widetilde{\boldsymbol{\mu}}=\ddot{\boldsymbol{\mu}}_{r}-\ddot{\boldsymbol{\mu}}+2 \boldsymbol{\Lambda} \dot{\tilde{\mu}}+\boldsymbol{\Lambda}^{2} \widetilde{\boldsymbol{\mu}} \\
& \dot{\tilde{\eta}}=\dot{\eta}-\dot{\hat{\eta}}=\dot{\eta}-\Gamma \xi
\end{aligned}
$$

From the assumption 2, it is assumed that the lumped disturbance variations are bounded and slowly varying, i.e., $\dot{\eta} \approx 0$, therefore, and substituting the time derivative of the centralized PID vector from Eq. (24), the manipulator equations of motion from Eq. (14), the proposed control vector from Eq. (18) and other relations from Eq. (19), into the Eq. (23) and simplifying, it becomes,

$$
\dot{V}=-\xi^{T} \mathbf{K} \xi
$$

Since, the controller and observer gain matrices $\mathbf{K}$ and $\boldsymbol{\Gamma}$ are constant symmetric and positive definite matri- ces, by design. It can be observed from Eqs. (21) and (26) that the Lyapunov's candidate function is positive definite and its time derivative is negative semidefinite in the entire state space. In order to prove the asymptotically convergence of the errors to zero, let consider a set $\boldsymbol{\Omega}$, and it contains of all points where $\dot{V}=0$, as follows:

$\boldsymbol{\Omega}=\left\{\zeta \in \Re^{6 \times 1} \mid \dot{V}=0\right\}$

The set $\boldsymbol{\Omega}$, is satisfied by $\boldsymbol{\Omega}=\left\{\zeta \in \mathfrak{R}^{6 \times 1} \mid \boldsymbol{\xi}=0\right\}$. If $\boldsymbol{\xi}(t)=0$, then $\ddot{\boldsymbol{\mu}}=0$. This implies that no solution can stay identically in $\boldsymbol{\Omega}$ other than $\widetilde{\boldsymbol{\eta}}(t)=0$. Therefore, based on Lyapunov's direct method and Barbalat's lemma (Kelly et al., 2005; Slotine and Li, 1991), the closed-loop system is asymptotically stable. i.e., the task-space position and velocity tracking errors, and the observer estimation errors are converging to zero 
asymptotically. i.e.,

$$
\begin{aligned}
& \lim _{t \rightarrow \infty} \boldsymbol{\xi}(t)=0, \lim _{t \rightarrow \infty} \tilde{\boldsymbol{\eta}}(t)=0, \\
& \lim _{t \rightarrow \infty} \dot{\tilde{\boldsymbol{\mu}}}(t)=0, \lim _{t \rightarrow \infty} \tilde{\boldsymbol{\mu}}(t)=0 .
\end{aligned}
$$

Therefore the manipulator follows the given desired task-space trajectory with minimal errors.

Remark 1: If the lumped disturbance term is fast varying, i.e., $\dot{\eta} \neq 0$ then a sufficient condition for the Lyapunov function derivative $\dot{V}$ in Eq. (26) to be negative semi-definite, is given as

$\dot{V}=-\xi^{T} \mathbf{K} \xi+\widetilde{\eta}^{T} \boldsymbol{\Gamma}^{-1} \dot{\eta}$

$\widetilde{\boldsymbol{\eta}}^{T} \boldsymbol{\Gamma}^{-1} \dot{\boldsymbol{\eta}} \geq 0$

where, $\boldsymbol{\Gamma}$ is the observer gain matrix and it is a constant symmetric and positive definite matrix by design, and by proper choice of this matrix can always satisfy the negative definite condition. In worst condition, value of $\widetilde{\boldsymbol{\eta}}^{T} \boldsymbol{\Gamma}^{-1} \dot{\boldsymbol{\eta}}>0$ is a small positive scalar. Then the Lyapunov function is a non-negative constant, such that $V(t) \rightarrow l$ as $t \rightarrow \infty$. Furthermore, $V(t) \leq V(0)$ and its derivative function is a negative definite (Kelly et al., 2005; Slotine and Li, 1991). In turn the observer errors, $\widetilde{\boldsymbol{\eta}}(t) \rightarrow 0$ as $t \rightarrow \infty$. In this case, the tracking controller and observer errors can be minimized arbitrarily by appropriate choice of design parameters (controller gain and observer gain matrices) and the uniform ultimate boundedness is guaranteed.

\section{Performance evaluation of the manipulator}

\subsection{Optimization of geometrical parameters for a given workspace}

This subsection presents the optimized geometrical design parameters namely link length, size of the mobile platform and the maximum limit of linear actuator stroke length of the manipulator for a given constant orientation workspace. For better understanding, the given workspace is chosen such that all the end-effector orientations are zero and three different cubical workspaces are considered for the optimization. The optimization (minimum) of the geometrical design parameters of the manipulator to reach all the points of a given workspace is considered. The optimization problem is carried subject to the following limits of the design parameters.

$$
\begin{aligned}
& 0 \mathrm{~m}<d_{i(\max )}<1 \mathrm{~m}, 0.1 \mathrm{~m}<l_{\mathrm{i}}<0.8 \mathrm{~m} \\
& \text { and } 0.04 \mathrm{~m}<r_{\mathrm{i}}<0.4 \mathrm{~m} .
\end{aligned}
$$

The foregoing optimization problems are solved by one of the popular optimization method namely genetic algorithms due to its search method and simplicity. Further, the numerical computation is solved by using the Matlab

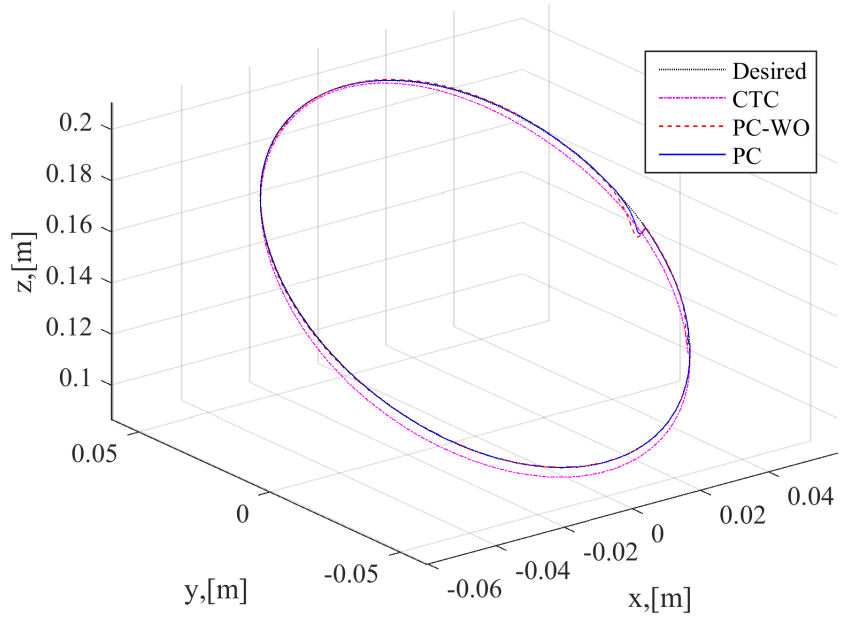

Figure 5. Block diagram representation of the proposed robust task-space control scheme.

genetic algorithms solver namely " $\boldsymbol{g} \boldsymbol{a}$ " function (with default settings) along with scanning method of the given workspace points. There are three cubical work volumes considered for the analysis namely $0.2 \mathrm{~m} \times 0.2 \mathrm{~m} \times 0.2 \mathrm{~m}$, $0.3 \mathrm{~m} \times 0.3 \mathrm{~m} \times 0.3 \mathrm{~m}$, and $0.4 \mathrm{~m} \times 0.4 \mathrm{~m} \times 0.4 \mathrm{~m}$. The optimized geometrical design parameters are given in Table 1. Based on these parameters, the virtual prototypes are developed in MSC Adams and performed the trajectory tracking control performance experiments.

\subsection{Description of the virtual prototype and the task}

Virtual prototype experiments are fulfilled to verify the effectiveness of the proposed control scheme. The parameters of the virtual prototype/simulation model are taken from the motion platform in the six-DOF vehicle simulation is being built in our own laboratory and their physical values are calculated by the aid of computer-aided design (CAD) models and their numerical values are given in Table 2 . The effectiveness of the proposed controller in following a given desired task-space trajectory in the presence of internal and external disturbances is validated by simulating the task of tracking a circular trajectory in 3-D space. The desired circular trajectory for the simulation is mathematically given as:

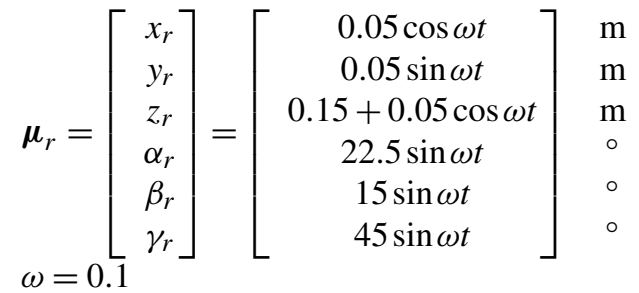

Although in this work, it is considered a circular trajectory for the analysis, in most of the robotic applications, smooth jerk-free motions in minimal time are desirable. Hence, these 


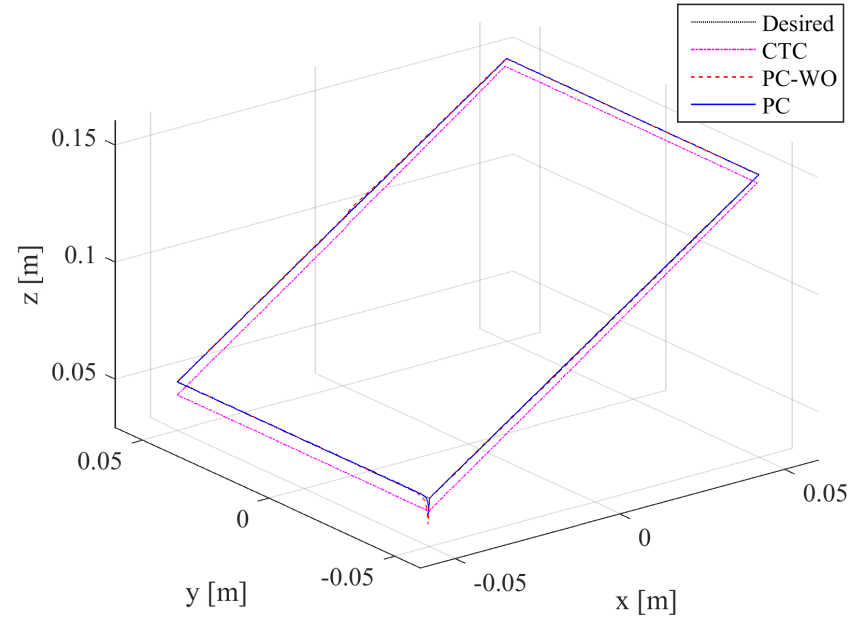

Figure 6. Block diagram representation of the proposed robust task-space control scheme.

criteria have to be considered during trajectory planning for a robotic system, wherein polynomial functions are often used for interpolating the trajectory through several via points. Among various polynomial functions, the cubic polynomial is the lowest degree polynomial that can provide a trajectory with $\mathrm{C}^{2}$ smoothness, which guarantees continuous acceleration. Therefore, cubic polynomials are chosen for a polygon trajectory generation and tracking task. Further, the endeffector orientations are assumed to be zero for simplicity. The desired polygon trajectory is mathematically given as:

$$
\begin{aligned}
& x_{r}= \\
& \left\{\begin{array}{c}
-0.05 \\
-0.05+4.8 \times 10^{-3}(t-25)^{2}-1.28 \times 10^{-5}(t-25)^{3} \\
0.05 \\
0.05-4.8 \times 10^{-3}(t-75)^{2}+1.28 \times 10^{-5}(t-75)^{3} \\
-0.05
\end{array}\right.
\end{aligned}
$$$$
\begin{array}{cc}
\mathrm{m} & 0 \leq t \leq 25 \\
\mathrm{~m} & 25<t \leq 50 \\
\mathrm{~m} & 50<t \leq 75 \\
\mathrm{~m} & 75<t \leq 100 \\
\mathrm{~m} & 100<t
\end{array}
$$

$$
\begin{aligned}
& y_{r}= \\
& \left\{\begin{array}{c}
-0.05+4.8 \times 10^{-3} t^{2}-1.28 \times 10^{-5} t^{3} \\
0.05 \\
0.05-4.8 \times 10^{-3}(t-50)^{2}+1.28 \times 10^{-5}(t-50)^{3} \\
-0.05 \\
-0.05
\end{array}\right.
\end{aligned}
$$$$
\text { m } 0 \leq t \leq 25
$$$$
\mathrm{m} \quad 25<t \leq 50
$$$$
\text { m } 50<t \leq 75
$$$$
\text { m } 75<t<100
$$$$
\mathrm{m} \quad 100<t
$$

$$
\begin{aligned}
& z_{r}= \\
& \left\{\begin{array}{ccc}
0.05 & \mathrm{~m} \quad 0 \leq t \leq 25 \\
0.05+4.8 \times 10^{-3}(t-25)^{2}-1.28 \times 10^{-5}(t-25)^{3} & \mathrm{~m} \quad 25<t \leq 50 \\
0.15 & \mathrm{~m} \quad 50<t \leq 75 \\
0.15-4.8 \times 10^{-3}(t-75)^{2}+1.28 \times 10^{-5}(t-75)^{3} & \mathrm{~m} & 75<t \leq 100 \\
0.05 & \mathrm{~m} & 100<t
\end{array}\right.
\end{aligned}
$$

$$
\alpha_{r}=0^{\circ}, \beta_{r}=0^{\circ}, \gamma_{r}=0^{\circ} \quad \forall t
$$

In order to analyse the controller robustness, process and measurement noises are added in the form of Gaussian noises during the performance analysis. Similarly, an unknown external disturbance vector has been considered and incorporated in the simulations; it is a kind of random slowly varying vector. For this analysis, it is assumed the estimated parameters are only $90 \%$ accurate with respect to the actual value. In addition, the manipulator initial velocities were set zero (start from rest) and the estimated system vectors were also considered as zero, while the initial desired and actual positions and orientations were assumed to be the same. The virtual prototype (simulation) model of the proposed manipulator in the Simulink background is shown in Fig. 4 and is integrated (co-simulation) with the MSC Adams model of the same manipulator.

\subsection{Simulation results and discussions}

In this subsection, virtual prototype experiment results for the above-mentioned tasks are presented and discussed to investigate the effectiveness and robustness of the proposed control scheme, which is expected to provide an intuitive, promising prospective of the proposed approach. To show the performance capability of the proposed robust control scheme, it is compared with other well-known scheme called a computed torque control (CTC) and is given by:

$$
\boldsymbol{\tau}_{\mathrm{c}}=\hat{\mathbf{M}}(\mu)\left[\ddot{\boldsymbol{\mu}}_{r}+\mathbf{K}_{P} \tilde{\boldsymbol{\mu}}+\mathbf{K}_{\mathrm{D}} \dot{\tilde{\boldsymbol{\mu}}}\right]+\hat{\mathbf{C}}(\mu, \dot{\mu}) \dot{\boldsymbol{\mu}}+\hat{\boldsymbol{g}}(\mu)
$$

In order to understand the disturbance observer role, the controller performances further compared with and without the presence of the disturbance observer. The results of the controller performance analysis done in a virtual prototype are presented in Figs. 5, 6 and 7. The performances of different controllers are abbreviated as follows: the computed torque control is abbreviated as CTC, the proposed controller with and without disturbance observers are abbreviated as PC and PCWO. The task-space position trajectories of a circular path and a polygon path are presented in Figs. 5 and 6, respectively. The time trajectories of the norm of tracking errors are presented in Fig.7. So as to understand the performance of the controller in a more quantitative way, the root-meansquare error analysis is performed by varying the controller parameters and working conditions. The root-mean-square (RMS) values of the vector of tracking errors of the endeffector pose are used as a performance measure quantity for the controller comparison and mathematically, it is given as:

$$
\begin{aligned}
\kappa_{\mathrm{rms}} & =\sqrt{\frac{\sum_{i=1}^{n}\left(x_{r i}-x_{i}\right)^{2}+\left(y_{r i}-y_{i}\right)^{2}+\left(z_{r i}-z_{i}\right)^{2}}{n}} \\
\varphi_{\mathrm{rms}} & =\sqrt{\frac{\sum_{i=1}^{n}\left(\alpha_{r i}-\alpha_{i}\right)^{2}+\left(\beta_{r i}-\beta_{i}\right)^{2}+\left(\gamma_{r i}-\gamma_{i}\right)^{2}}{n}}
\end{aligned}
$$

where, $\kappa_{\text {rms }}$ is the RMS value of end-effector position errors and $\varphi_{\mathrm{rms}}$ is the RMS value of end-effector orientation errors.

From these results, it is found that the tracking performance is improved when the proposed control scheme applied to the manipulator. The values of the tracking errors 


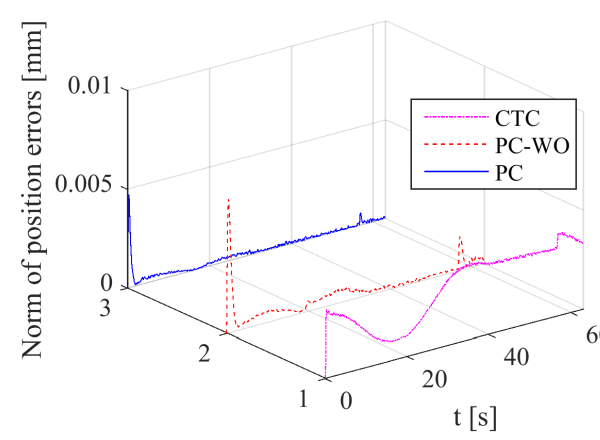

(a) Position errors for the Circular path

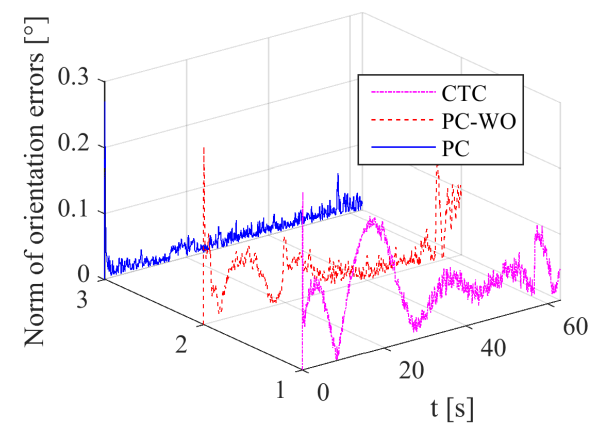

(c) Orientation errors for the Circular path

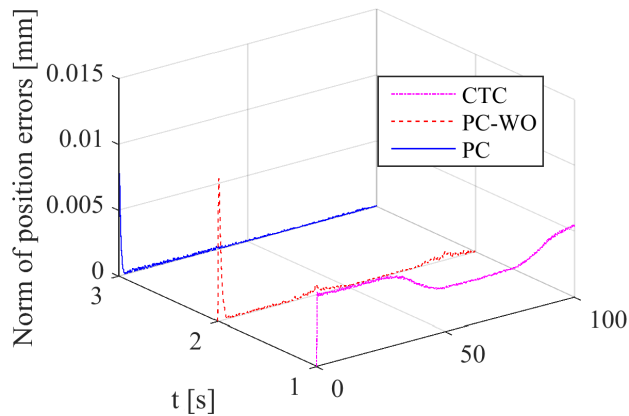

(b) Position errors for the Polygon path

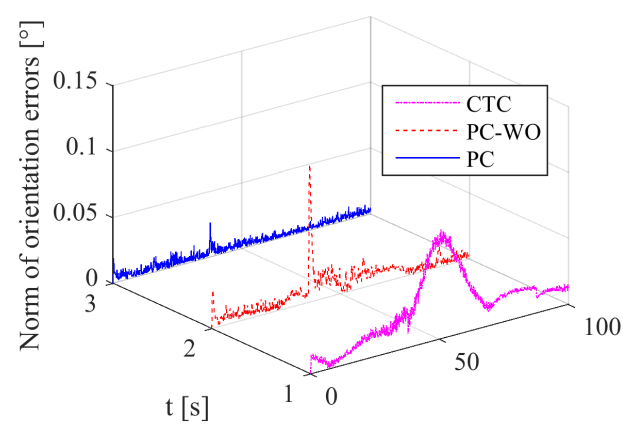

(d) Orientation errors for the Polygon path

Figure 7. Time trajectories of the norm of tracking errors.
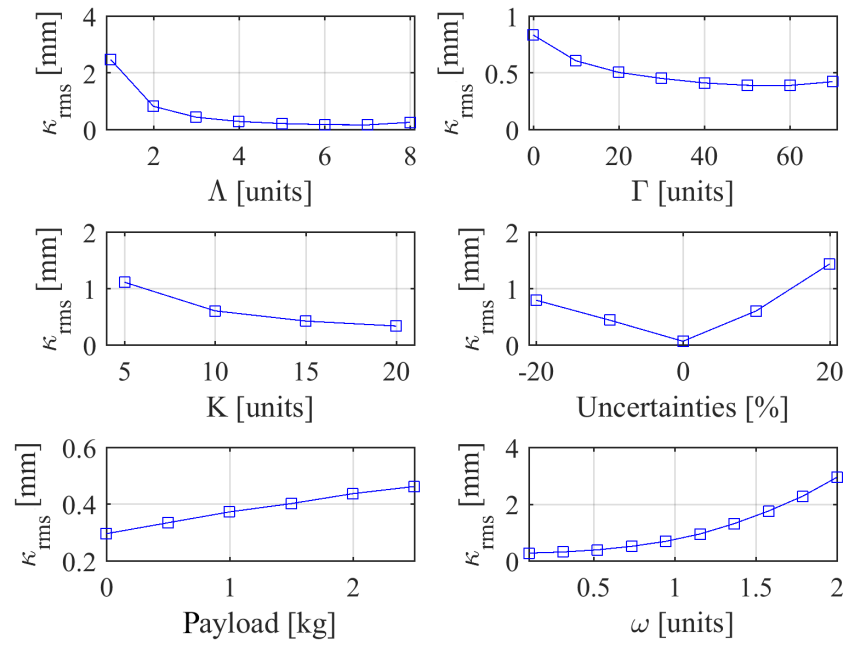

Figure 8. Controller parameter sensitivity and robustness analysis results: Parameter variations vs. Root-mean-square error of endeffector positions.

are high at the initial stage which is due to the presence of zero observer values and non-zero gravity compensation (presence of $\Delta \boldsymbol{g}(\mu)=\boldsymbol{g}(\mu)-\hat{\boldsymbol{g}}(\mu)$ ). However, over a period of time, the proposed controller compensates this effect due to the centralized PID control vector and the disturbance observer. But in the case of computed torque control, this effect could remain and because of the only PD con-
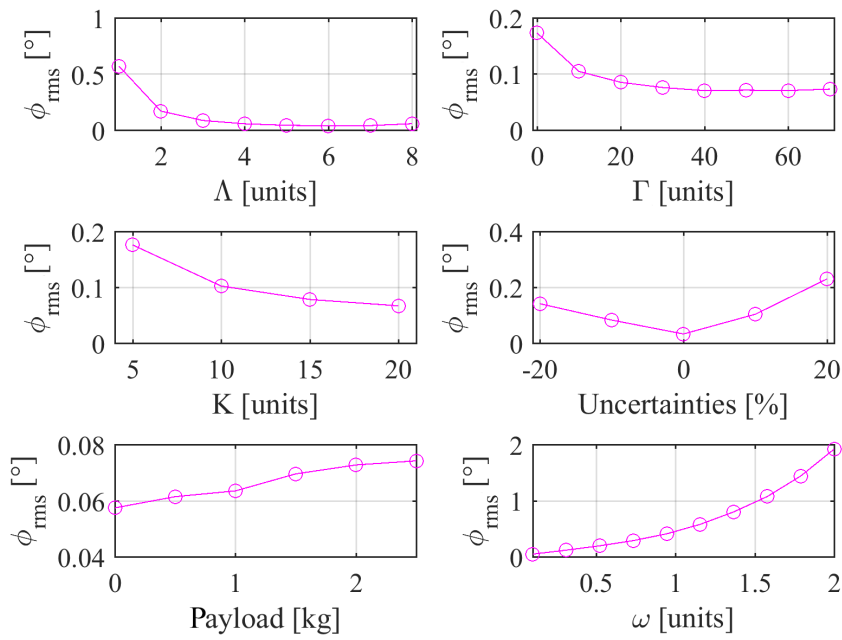

Figure 9. Controller parameter sensitivity and robustness analysis results: Parameter variations vs. Root-mean-square error of endeffector orientations.

trol action, the steady state error exists in the performance. The RMS values of end-effector position errors for the CTC, proposed controller without and with disturbance observer for the circular tracking are 3.02, 0.84 and $0.43 \mathrm{~mm}$, respectively. That is, 85 and $49 \%$ improvement in the average RMS values is observed during circular trajectory tracking from the CTC scheme and proposed controller without disturbance 

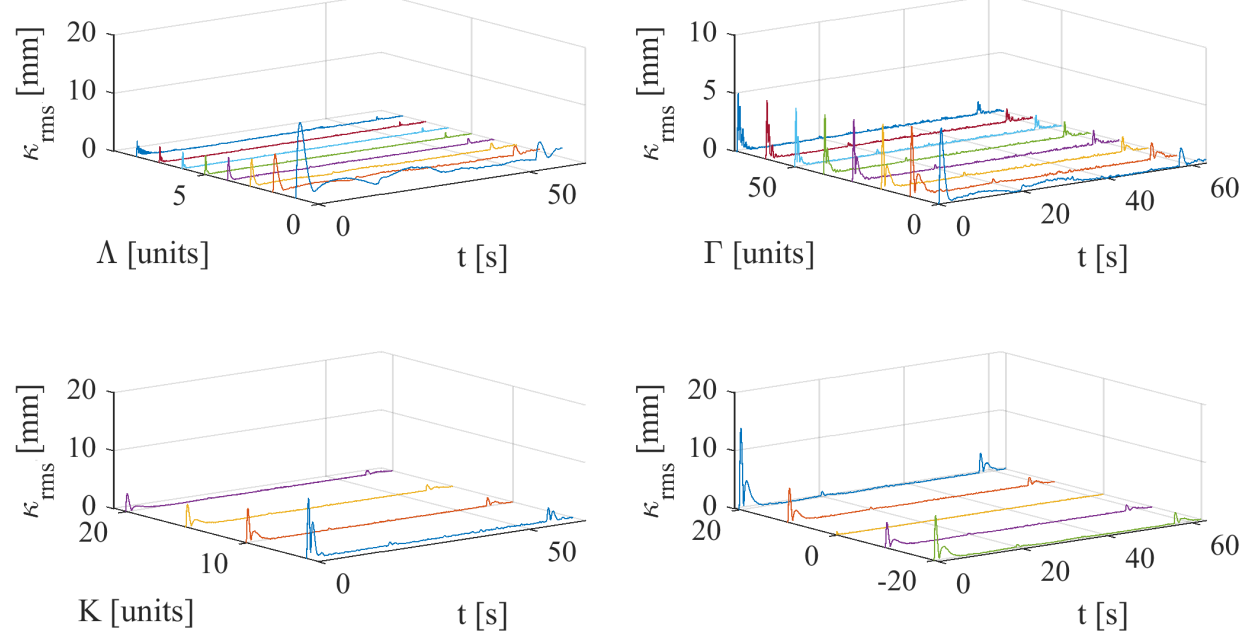

Uncertainties [units]
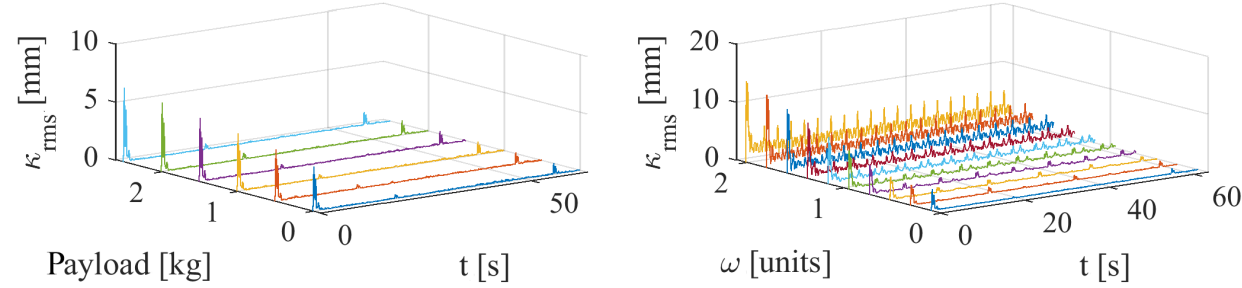

Figure 10. Sensitivity and robustness analysis results: Time trajectories of the norm of the end-effector position errors.
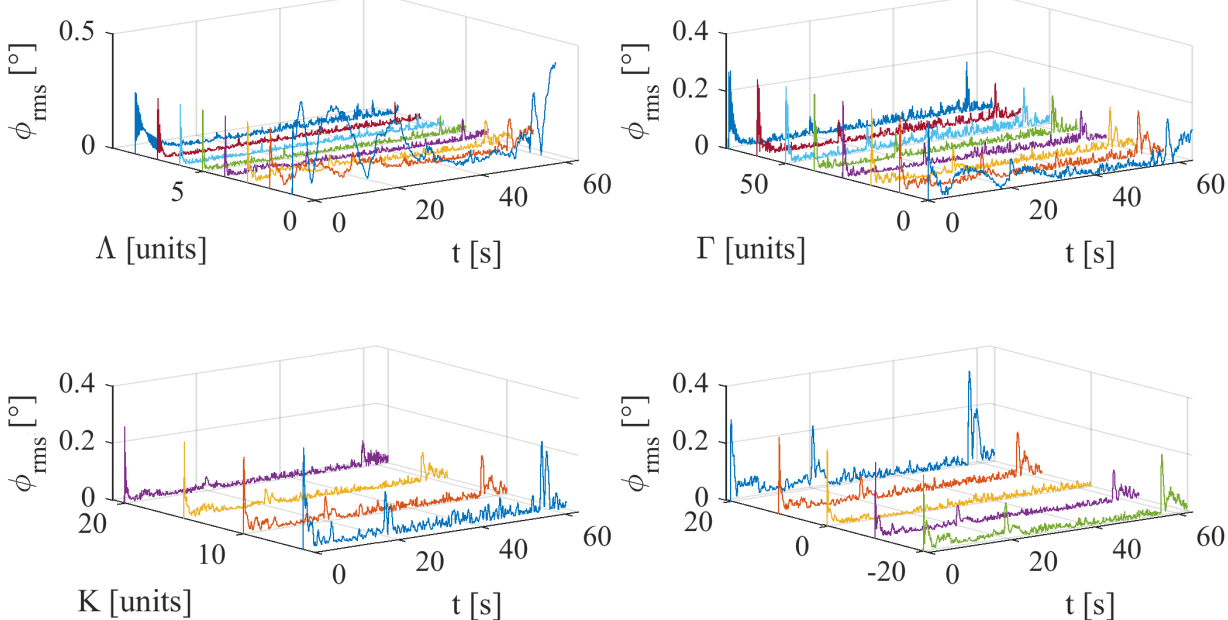

Uncertainties,[units]
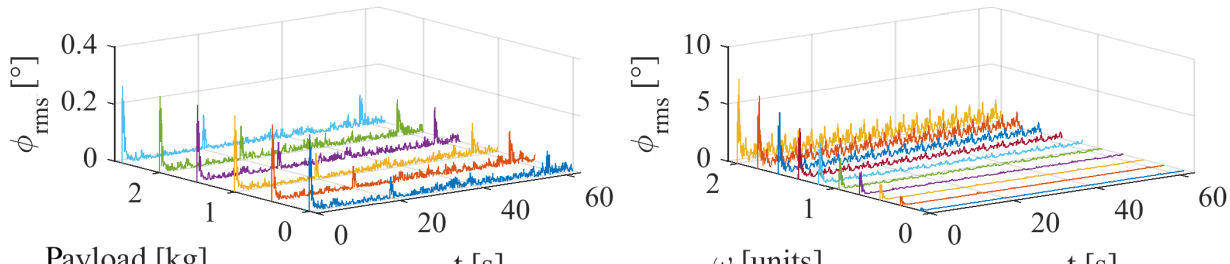

Payload $[\mathrm{kg}]$ $\mathrm{t}[\mathrm{s}]$

$\omega$ [units]

$\mathrm{t}[\mathrm{s}]$

Figure 11. Sensitivity and robustness analysis results: Time trajectories of the norm of the end-effector orientation errors. 
observer by applying the proposed controller. The same trend is approximately followed in the polygon path as well. From the root mean square error analysis of orientation, it is found that the proposed controller reduced the end-effector orientation errors to $0.05^{\circ}$ as compared to other controllers. Since the desired end-effector orientations are zero in the case of polygon/cubic polynomial path, the differences in the orientation errors of all three controllers are very less.

Further to understand the robustness and behaviour of the proposed control scheme when there are variations in its controller parameters, payload, uncertainty and dynamic working conditions, the robustness and sensitivity analyses are conducted by tracking a circular trajectory of the end-effector positions as mentioned earlier with different operating conditions. Apart from varying the controller parameters, the working conditions are also varied in this analysis.

The percentage of parameter uncertainties is varied from -20 to $+20 \%$, i.e., the gravity vector, inertia and Coriolis matrices are inaccurately known with these percentage variations. Similarly, for the payload variations, an additional mass added to the end-effector and the value of the mass is varied from 0 to $2.5 \mathrm{~kg}$. In addition, the frequency of the circular trajectory as mentioned in Eq. (32), $\omega$ value is also varied 0.1 to 2 , instead of keeping a constant value as 0.1 . The controller parameter sensitivity and robustness analysis results are presented in Figs. 8-11. The RMS values of end-effector position and orientation errors are plotted in Figs. 8 and 9, respectively. Similarly, time histories of the end-effector position and orientation errors are plotted in Figs. 10 and 11 respectively. These plots show that the error variations are very minimal for the parameter variations, expect at higher speeds of operations. From overall results, it is observed that the proposed controller is robust enough for the parameter uncertainties, un-modelled dynamics and payload variations.

\section{Conclusions}

In this paper, the inverse dynamic model and a robust adaptive motion control scheme of a 6-DOF spatial 3-RPRS parallel manipulator system in the presence of parametric uncertainties and external disturbances have been investigated. The proposed control strategy was designed to track the given desired end-effector trajectory in the task-space with minimal errors. The effectiveness of the proposed controller was verified by simulation. From the obtained numerical simulation results, the strength of proposed control scheme can be summarized as follows:

- Proposed controller increases the overall stability of closed loop system as compared to conventional controllers.

- Poor knowledge of the system parameters will be sufficient to design the controller.

- Proposed control scheme provides great immunity to the external disturbances and parameter uncertainties as compared to conventional controllers.

- Proposed controller has simple control structure and design. Hence, it can be used for time implementation with a low-cost microprocessor.

- The proposed controller can also be applied to other kinds of parallel manipulators.

Future work will concentrate on the real implementation of the proposed controller to our in-house fabricated manipulator prototype system. Also, in upcoming studies, the evaluation shall be made between the proposed scheme and the latest published studies for this kind of manipulator to examine whether this technique is robust when compared with new and recent controllers and the well-tuned proportional controller with the feedforward compensation.

Data availability. All the data used in this manuscript can be obtained by requesting from the corresponding author. 


\section{Appendix A: Nomenclature}

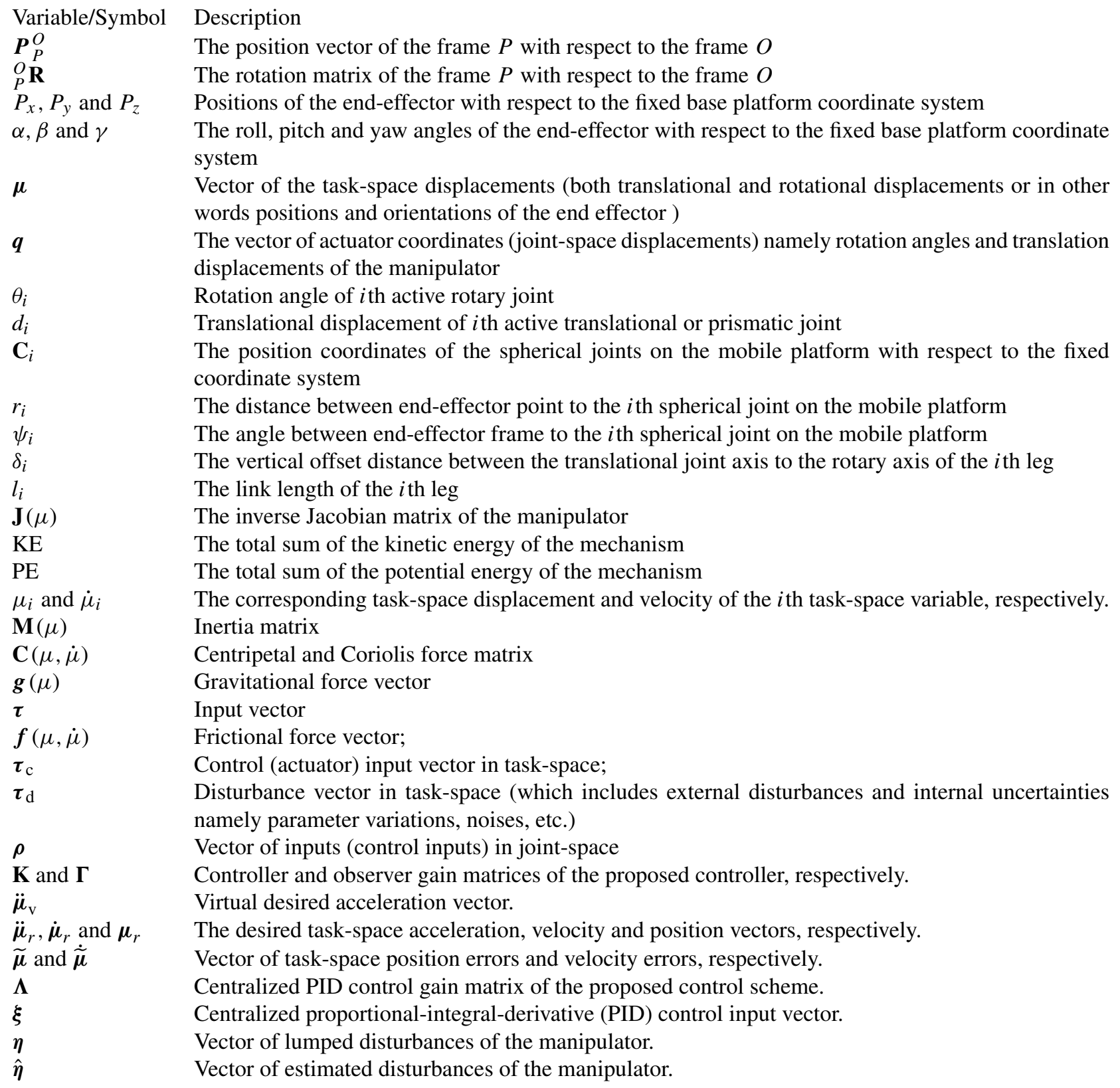


Competing interests. The authors declare that they have no conflict of interest.

Acknowledgements. The financial support of the first author as a Humboldt Research Fellow by the Alexander von Humboldt $(\mathrm{AvH})$ Foundation, Germany is gratefully acknowledged.

Edited by: Andreas Müller

Reviewed by: three anonymous referees

\section{References}

Abdellatif, H. and Heimann, B.: Computational efficient inverse dynamics of 6-DOF fully parallel manipulators by using the Lagrangian formalism, Mech. Mach. Theory, 44, 192-207, https://doi.org/10.1016/j.mechmachtheory.2008.02.003, 2009.

Alizade, R. I. and Tagiyev, N. R.: A forward and reverse displacement analysis of a 6-DOF in-parallel manipulator, Mech. Mach. Theory, 29, 115-124, https://doi.org/10.1016/0094114X(94)90024-8, 1994.

Bonev, I. A.: Analysis and design of 6-DOF 6-PRRS parallel manipulators, Master Thesis, Kwangju Institute of Science and Technology, Kwangju, 1998.

Briot, S., Arakelian, V., and Guégan, S.: PAMINSA: A new family of partially decoupled parallel manipulators, Mech. Mach. Theory, 44, 425-444, https://doi.org/10.1016/j.mechmachtheory.2008.03.003, 2009.

Byun, Y. K. and Cho, H. S.: Analysis of a novel 6-DOF, 3-PPSP parallel manipulator, Int. J. Robot. Res., 16, 859-872, 1997.

Carbonari, L., Battistelli, M., Callegari, M., and Palpacelli, M.-C.: Dynamic modelling of a 3-CPU parallel robot via screw theory, Mech. Sci., 4, 185-197, https://doi.org/10.5194/ms-4-185-2013, 2013.

Dasgupta, B. and Mruthyunjaya, T.: The Stewart platform manipulator: a review, Mech. Mach. Theory, 35, 15-40, https://doi.org/10.1016/S0094-114X(99)00006-3, 2000.

Davliakos, I. and Papadopoulos, E.: Model-based control of a 6-dof electrohydraulic Stewart-Gough platform, Mech. Mach. Theory, 43, 1385-1400, https://doi.org/10.1016/j.mechmachtheory.2007.12.002, 2008.

Honegger, M., Brega, R., and Schweizer, G.: Application of a nonlinear adaptive controller to a 6 DOF parallel manipulator, in: Proceeding of the 2000 IEEE International Conference on Robotics and Automation, San Francisco, 2000.

Isaksson, M., Brogårdh, T., Watson, M., Nahavandi, S., and Crothers, P.: The Octahedral Hexarot - A novel 6-DOF parallel manipulator, Mech. Mach. Theory, 55, 91-102, https://doi.org/10.1016/j.mechmachtheory.2012.05.003, 2012.

Kelly, R., Santibanez, V., and Loria, A.: Control of Robot Manipulators in Joint Space, Springer, London, UK, 2005.

Kim, D. H., Kang, J. Y., and Lee, K.-II.: Robust tracking control design for a 6 DOF parallel manipulator, J. Robotic Syst., 17, 527-547, https://doi.org/10.1002/10974563(200010)17:10<527::AID-ROB2>3.0.CO;2-A, 2000.
Kim, H. S., Cho, Y. M., and Lee, K.-II.: Robust nonlinear task space control for a 6 DOF parallel manipulator, Automatica, 41, 15911600, https://doi.org/10.1016/j.automatica.2005.04.014, 2005.

Liu, X. J., Wang, J., Gao, F., and Wang, L. P.: Mechanism design of a simplified 6-DOF 6-RUS parallel manipulator, Robotica, 20, 81-91, https://doi.org/10.1017/S0263574701003654, 2002.

Merlet, J.-P.: Parallel Robots, Kluwer Academic Publishers, Boston, 2000.

Nag, A., Mohan, S., and Bandyopadhyay, S.: Forward kinematic analysis of the 3-RPRS parallel manipulator, in: New Trends in Mechanism and Machine Science: Theory and Industrial Applications, edited by: Wenger, P. and Flores, P., Springer International Publishing, 103-111, https://doi.org/10.1007/978-3-31944156-6, 2017.

Nguyen, A. V., Bouzgarrou, B. C., Charlet, K., and Béakou, A.: Static and dynamic characterization of the 6-Dofs parallel robot 3CRS, Mech. Mach. Theory, 93, 65-82, https://doi.org/10.1016/j.mechmachtheory.2015.07.002, 2015.

Nguyen, C. C., Antrazi, S. S., Zhou, Z. L., and Campbell, C. E.: Adaptive control of a Stewart platformbased manipulator, J. Robotic Syst., 10, 657-687, https://doi.org/10.1002/rob.4620100507, 1992.

Pierrot, F.: A new design of a 6-DOF parallel robot, Journal of Robotics and Mechatronics, 2, 308-315, https://doi.org/10.20965/jrm.1990.p0308, 1990.

Ryu, S. J., Kim, J., Hwang, J., Park, C., Kim, J., and Park, F. C.: ECLIPSE: An Overactuated Parallel Mechanism for Rapid Machining, in: Proceedings of the 12th CISM-IFTOMM Symposium on the Theory and Practice of Robots and Manipulators, Paris, 1998.

Slotine, J. J. E. and Li, W.: Applied Nonlinear Control, PrenticeHall, London, 1991.

Tahmasebi, F. and Tsai, L-W.: Six-degree-of-freedom Parallel "Minimanipulator" With Three Inextensible Limbs, U.S. Patent No. 5,279,176, 1994.

Ting, Y., Chen, Y. S., and Jar, Y. S.: Modeling and control for a Gough-Stewart platform CNC machine, J. Robotic Syst., 21, 609-623, https://doi.org/10.1002/rob.20039, 2004.

Uchiyama, M.: A 6 d.o.f. parallel robot HEXA, Adv. Robotics, 8, 601-601, https://doi.org/10.1163/156855394X00293, 1993.

Venkatesan, V., Singh, Y., and Mohan, S.: Inverse Kinematic Solution of a 6-DOF (3-RPRS) Parallel Spatial Manipulator, in: Proceedings of the 3rd Joint International Conference on Multibody System Dynamics - IMSD 2014, Busan, 2014.

Wu, D. and Gu, H.: Adaptive Sliding Control of Six-DOF Flight Simulator Motion Platform, Chinese J. Aeronaut., 20, 425-433, https://doi.org/10.1016/S1000-9361(07)60064-8, 2005.

Yang, C., Huang, Q., Jiang, H., Peter, O.O., and Han, J.: PD control with gravity compensation for hydraulic 6-DOF parallel manipulator, Mech. Mach. Theory, 45, 666-677, https://doi.org/10.1016/j.mechmachtheory.2009.12.001, 2010.

Zhang, D.: Parallel Robotic Machine Tools, Springer, London, https://doi.org/10.1007/978-1-4419-1117-9, 2010. 